Grand Valley State University

ScholarWorks@GVSU

\title{
Application of Cognitive-Behavioral Treatment for Long-Standing Posttraumatic Stress Disorder in Law Enforcement Personnel
}

Tara L. Cornelius

Grand Valley State University, cornelta@gvsu.edu

Rita Kenyon-Jump

Veterans Affairs Medical Center

Follow this and additional works at: https://scholarworks.gvsu.edu/psy_articles

Part of the Psychology Commons

\section{ScholarWorks Citation}

Cornelius, Tara L. and Kenyon-Jump, Rita, "Application of Cognitive-Behavioral Treatment for LongStanding Posttraumatic Stress Disorder in Law Enforcement Personnel" (2007). Peer Reviewed Articles. 53.

https://scholarworks.gvsu.edu/psy_articles/53

This Article is brought to you for free and open access by the Psychology Department at ScholarWorks@GVSU. It has been accepted for inclusion in Peer Reviewed Articles by an authorized administrator of ScholarWorks@GVSU. For more information, please contact scholarworks@gvsu.edu. 


\title{
Application of Cognitive-Behavioral Treatment for Long-Standing Posttraumatic Stress Disorder in Law Enforcement Personnel
}

\section{A Case Study}

\author{
Tara L. Cornelius \\ Grand Valley State University, Allendale, Michigan \\ Rita Kenyon-Jump \\ Veterans Affairs Medical Center, Battle Creek, Michigan
}

\begin{abstract}
The development and maintenance of posttraumatic stress disorder (PTSD) can be understood as a function of learning and cognitive processes. This case report addresses treatment of PTSD with a 72-year-old former police officer who was experiencing intrusive thoughts, nightmares, experiential and affective avoidance, and hyperarousal resulting from witnessing a series of traumatic events during his time in law enforcement. Although the latency between events and treatment was more than 20 years, this patient was responsive to a cognitive-behavioral, exposure-based treatment. Several other factors, including the client's age and chronic pain, complicated this case. The aim of this study was to identify the conceptual framework for treatment, explore the course of therapy, and identify socially valid means of treatment and assessment in outpatient therapy. At the termination of treatment, this patient evidenced improvements in overall anxiety, depressive symptoms, and frequency and severity of PTSD symptoms. Recommendations for clinicians and treatment implications are also presented.
\end{abstract}

Keywords: PTSD; CBT; exposure treatments

\section{Theoretical and Research Basis}

The development and maintenance of posttraumatic stress disorder (PTSD) can be understood as a function of conditioning and cognitive processes. Learning theories, which include operant and classical conditioning, emphasize the importance of previous learning trials with the feared stimuli and the role of avoidance conditioning in the maintenance of the phobia or fears. In contrast, cognitive approaches examine and focus on the role of beliefs, core assumptions, and evaluative processes that relate to the stimulus events and contend that these factors play a critical role in development and maintenance of pathological anxiety and fear that characterize anxiety disorders as a diagnostic taxonomy. Cognitive-behavioral approaches to treating anxiety disorders, then, incorporate the theoretical underpinnings and therapeutic approaches from both these traditions, to treat psychopathology from numerous points of entry (Foa, 1996; Smyth, 1999). 
Behavioral theories of anxiety reduction were developed, in large part, from the theoretical perspective known as the two-factor theory of learning, pioneered by Mowrer (1960). According to this theory, the development of pathological anxiety develops via classical conditioning operations, and maintenance and avoidance learning is facilitated by operant conditioning processes. Fear acquisition occurs through the process of classical conditioning, in which a formerly neutral stimulus (the conditioned stimulus), following a pairing with an aversive unconditioned stimulus, comes to elicit the fear response, the conditioned response. Although classical conditioning operations result in the development of pathological anxiety, operant conditioning processes facilitate the maintenance of anxiety responses. Operant conditioning examines the consequences of events as determinants of behavior. In the case of PTSD, individuals learn to avoid or escape trauma-related anxiety because it is an aversive state; that is, avoidance or escape from trauma-related cues is reinforced by the resulting reduction in anxiety or fear response and the associated physiological arousal. Escape and avoidance behaviors become established and maintained through the process of negative reinforcement, in that such behaviors terminate the aversive fear state.

Cognitive approaches to treating and conceptualizing anxiety disorders utilize mechanisms to identify, understand, and modify maladaptive cognitive processes, including but not limited to beliefs, core assumptions, and evaluative processes that relate to the stimulus events of the trauma. These cognitive and mental collateral responses not only are present in the initial behavioral pairing during the trauma but also play a critical role in the maintenance of the anxiety response. For example, believing that the event is one's fault, that one is to blame, or the continuous process of ruminating and reliving the events can serve as secondary trauma and continue to the process of pathological anxiety (Smyth, 1999).

\section{Case Presentation}

Mr. Jones (name changed to preserve confidentiality) was a 72-year-old, married African American male who was referred by his civilian general practitioner to a VA Medical Center outpatient mental health clinic. He had never participated in any inpatient or outpatient mental health services. Mr. Jones had been married for 40 years and reported that his marital relationship was satisfying, although his wife would like him to disclose more about his trauma history. He had three grown children.

Mr. Jones served as a police officer for several years in a moderately sized city and was involved in and witnessed three events, in close chronology, that resulted in the death of other individuals. These events occurred nearly 30 years prior to beginning treatment and resulted in chronic distress and symptoms since that time. He stated that all of the incidents involved high-speed chases, one resulted in him doing cardiopulmonary resuscitation (CPR) on an 11-year-old boy who died at the hospital, one resulted in the death of all the passengers in the other car, and the last involved a similar accident, although there were no fatalities. He stated that for several years after the events, he did not drive past places where the incidents occurred. At the time of these incidents, his police agency did not have counseling for officers who experienced traumatic events such as he described. He reported that he had never discussed the details of these events with anyone since they occurred and often attempted to hide or minimize his distress in the presence of others. 


\section{Presenting Complaints}

The patient reported symptoms of avoidance, intrusive thoughts, flashbacks, and past mood disturbances resulting from the incidents in the early 1970s. One of the events involved a high-speed chase, which resulted in the death of the driver and his passengers. In addition, he stated that less than 1 year after the first event, he was involved in another chase, at the same intersection, which resulted in the death of the passenger in the car: an 11-year-old child. He was not found to be at fault in these incidents, although he was transferred from the evening to day shift, and continued to feel guilt and responsibility for these events. At the onset of treatment, he continued to have difficulty driving on two-lane highways and passing the site of the accidents and reported flashbacks and hyperarousal when presented with similar stimuli. He stated that on many occasions he "can't stop thinking about the images" associated with the events and reported that on occasion, he could "hear, see, and smell" the traumatic events. He stated that on some occasions when he experienced intrusive thoughts from these events his family noticed that he "clams up" and socially isolates himself. At onset of treatment, he reported regular nightmares and flashbacks of incidents, particularly those stimuli relating to administering emergency treatment to the child. He reported depressed mood, social isolation, and sadness resulting from these events.

He also reported that 9 years following these events, he was injured on the job and continues to experience chronic daily pain resulting from these injuries. He has had several surgeries to deal with the pain but continues to report pervasive neck, back, and leg pain. He stated that his physical pain serves as "a constant reminder of those events." These injuries resulted in him being unable to serve in the same capacity on the police force, and he was offered several different administrative duties, which he found unsatisfying. He noted during the interview, "This was devastating, horrible. I loved serving on the police force, and I lost it. This kills me."

\section{History}

Mr. Jones reported no prior treatment for psychiatric or substance abuse problems. He reported that his current symptomatology had been ongoing for nearly 30 years, and that it has remained chronic and stable over that time. He denied any other psychiatric concerns and has never been prescribed or taken any psychotropic medication. He denied current suicidal or homicidal ideation and denied any history of suicidal ideation or intent. He denied familial history of mental illness, substance abuse, or suicidal or homicidal behavior.

Mr. Jones reported a positive relationship with his parents growing up. He stated that his family taught him "good family values." He is the first of seven siblings. His parents are deceased, and he reported regular, positive contact with all his siblings and noted that he has a strong extended family network. Mr. Jones knows of no developmental difficulties, learning difficulties, birth-related problems, or significant childhood illnesses or injuries that he experienced. He reported that he was an average to low-average student in grade school and high school. He noted typical peer relationships and interests in sports. Immediately following high school, he joined the Army. He served for 4 years in noncombat areas, after which he attended a community college and earned a certificate in criminal justice. 
Following his education, Mr. Jones served as a police officer for more than 20 years, during which time he witnessed several tragic and disturbing events, and was injured himself. He was assigned to regular patrol duties until his injury resulted in reassignment to administrative duties. He eventually retired from the police force and currently works part-time at a grocery store. Mr. Jones reports ongoing chronic, daily pain in his neck, shoulders, legs, and back.

\section{Assessment}

Prior to and during the course of treatment, several assessments were completed to determine current functioning and progress in treatment. Prior to beginning treatment, an abbreviated mental status examination was administered to determine current levels of cognitive and emotional functioning. During this initial evaluation, psychomotor retardation was apparent, as evidenced by reduced hand gestures or movement. However, this may have been because of the veteran's movement limitations from his physical pain. Mr. Jones maintained appropriate eye contact throughout the assessment. He denied hallucinations or delusions, and there was no evidence of psychotic processes. During the assessment, the patient was oriented to person, place, and time, and his speech was clear and goal directed. He was somewhat reserved and provided few details when questioned, although this lessened in future sessions. Attention and concentration appeared grossly within normal limits. He appeared to be of at least average intellectual abilities. He rated his pain at a 6, and there were behavioral signs of this pain, including wincing and repositioning himself in the chair.

Throughout the course of treatment, several measures were administered to determine the presence and progress of relevant anxiety, depressive, and PTSD-related symptoms. The first administration of the measures was completed during the first session following the intake interview and continued throughout treatment, occurring, on average, every other session. The following assessment instruments were used throughout treatment.

The Beck Depression Inventory 2nd edition (BDI-II; Beck, Steer, \& Brown, 1996) is a brief, self-report questionnaire consisting of 21 items. The items assess presence of various depressive symptoms during a 2-week period with four options under each item, ranging from 0 (not present) to 3 (severe). Individual item scores are summed to a total score that represented the overall severity of depressive symptomatology. The BDI-II has demonstrated good internal consistency, test-retest reliability, and concurrent validity (Steer, Ball, Ranieri, \& Beck, 1997; Waller, 1998).

The Beck Anxiety Inventory (BAI; Beck, Brown, Epstein, \& Steer, 1988) is a 21-item measure designed to assess common symptoms of anxiety, often occurring concurrently with PTSD. The respondent is asked to rate how much he or she has experienced each symptom over the past week on a 4-point scale ranging from 0 to 3 . The items are summed to obtain a total score that can range from 0 to 63 , which indicates the overall level of anxiety experienced. This scale has demonstrated strong test-retest reliability, internal consistency, and convergent and discriminant validities (Beck et al., 1988).

The Modified PTSD Symptom Scale (MPTSDSS; Falsetti, Resnick, Resick, \& Kilpatrick, 1992) is a 17-item self-report measure. This scale was explicitly designed to measure the frequency and severity of PTSD-related symptoms in the preceding 2 weeks. The frequency items are scored on a scale from 0 to 3, relating to the frequency of each 
PTSD symptom in the past week. Severity is measured on a 5-point Likert-type scale. The MPTSDSS has been validated on community and treatment populations and demonstrated good internal consistency and concurrent validity (Falsetti et al., 1992).

\section{Case Conceptualization}

Mr. Jones reported chronic, ongoing symptoms of PTSD resulting from three traumatic events occurring during his time as a police officer. These symptoms have negatively affected his functioning in terms of experiential avoidance, flashbacks, nightmares, and intrusive thoughts. In addition, these symptoms have had a lasting effect on his ability to form close interpersonal relationships, particularly with his wife and children. Although he reported generally satisfactory relationships with these individuals, he noted that he was reticent to disclose emotional experiences or discuss his traumatic history. His sense of loss and isolation is exacerbated by his chronic pain, which results from an injury sustained as a police officer. In addition to the loss of mobility, these injuries resulted in his inability to perform his regular duties as a police officer, which can be conceptualized as further loss. Subsequent to his symptoms of PTSD, in the past, depressive symptoms have occurred, although they were mild at the onset of treatment.

Since the time of the events, Mr. Jones had been utilizing strategies of avoidance, emotional and experiential, to deal with his PTSD symptoms. He had been avoiding the physical environments associated with the events, in addition to locales that evidenced some similarity with the original locations. These behaviors, while reducing or avoiding immediate anxiety, reinforced subsequent avoidance behavior, thus strengthening the anxiety response. This is not only contraindicated to improvements in his symptomatology, it also has resulted in functional impairments for Mr. Jones, in that his range of activities was diminished. Experiential avoidance is common in police officers with a traumatic history. Solomon (1992) found that separated police officers often constrict their scope of activity, social ties, and other civilian activities following traumatic exposures. It is interesting to note, Mr. Jones even appeared to be utilizing avoidance strategies while discussing the traumas during the first session. He reported the details of the events in a detached, formal fashion, as if he was reading from a police report; that is, while he could discuss the facts of the events, he was emotionally detached from the events. This is not necessarily surprising, given the training in detachment, rational thought, calmness, and objectivity inherent in police work, but presented some areas of challenge in treatment. This detachment was perceived by others in his life, notably his wife, who stated that she would like to share in his emotional experiences as well.

As is the case for younger adults, chronic anxiety and anxiety disorders lower life satisfaction and the quality of life of the patient (J. M. Cook, 2002; Wetherell, 1998). Treatments for anxiety in older adult populations have been demonstrated to be effective (Carmin \& Wiegartz, 2000; Maercker, 2002). In particular, relaxation training, which is one of the most common forms of treatment for anxiety symptoms, was demonstrated to be more effective than cognitive treatments for older adults (Wetherell, 1998). In addition, after comprehensively reviewing the literature on anxiety and adults, Wetherell (1998) found that the combination of relaxation, cognitive therapy, and behavioral strategies, especially those 
that utilize exposure, may be most effective for older adults. However, several considerations are important when working with older adults who are anxious. First, because medical issues may interfere or even masquerade as symptoms of anxiety, it is even more important than typical that these patients be closely monitored by their medical team and a psychiatrist. In addition, because of weakened health status and increased risk of cardiopulmonary complications, reduced treatment intensity is often recommended, especially when using techniques that induce high levels of subjective anxiety. Thus, when using exposure methods, anxiety levels should remain in the mild to moderate range, and gradual exposure is likely preferable over flooding methodology (Wetherell). In addition, in part because of the more gradual approach to treatment, the number of sessions required by older clients to become proficient in specific skill modules may be up to twice as long as the number required by younger clients (Wetherell).

These factors, in addition to the amount of time that transpired between the traumas and treatment, necessitate consideration in determining the course of treatment (A. L. Cook \& Shear, 2001). Given these factors, it is possible that Mr. Jones may have difficulty and possibly withdraw from an intensive or prolonged exposure treatment regime. In this case, a gradual and brief exposure would be a better approach, in terms of treatment outcomes and acceptability of the treatment to the patient. These treatments have the additional benefit of providing some techniques (i.e., relaxation training) that may improve his ability to cope with his chronic pain issues. Exposure-based treatments for PTSD have been empirically demonstrated to be effective for these patients (Foa \& Rothbaum, 1989; Smyth, 1999) and have been deemed as "probably efficacious" by the task force on empirically validated treatments (Chambless et al., 1998).

\section{Course of Treatment and Assessment of Progress}

Treatment consisted of 15 outpatient sessions occurring during the course of 7 months. Sessions had an average duration of approximately 60 minutes. The primary goals of treatment included reduction in the frequency and severity of PTSD symptoms, including experiential and cognitive avoidance, hyperarousal, social isolation, nightmares, and intrusive thoughts. In addition, it was hypothesized that developing anxiety-management skills may also be therapeutic for Mr. Jones in better managing and coping with his chronic pain. Exploration of the trauma in the context of a therapeutic relationship permits the client to reframe his beliefs about the trauma as an isolated event rather than something that defines his personal identity. The treatment process itself is often therapeutic in that it demonstrates to the client that he or is capable of mastery of the cognitive, behavioral, and physiological elements of the traumatic memories. Treatment design and implementation was guided by an empirically based protocol, Overcoming Post Traumatic Stress Disorder (Smyth, 1999), although the specific examples used and course of treatment was flexible to meet Mr. Jones' experiences and needs.

The initial stages of treatment (Sessions 1-3) focused on building rapport and introducing the rationale for and basic tenets of exposure-based treatments. Because exposure treatments are often emotionally strenuous, because they involve deliberate evocation of anxietyprovoking stimuli, developing a trusting relationship is essential to effective treatment and 
reduces the likelihood of premature attrition. During the initial sessions, it appeared that Mr. Jones was having difficulty trusting and relating to the therapist, as evidenced by reticence to divulge details, reporting his symptoms in a detached, formalized fashion, and limited eye contact. As part of the initial sessions, the rationale for exposure-based treatments was provided, including the nature of eliciting traumatic memories and symptoms. To adequately prepare Mr. Jones for the treatment and normalize any PTSD symptoms that he may have experienced, he read a brief psychoeducational description of cognitive-behavioral treatment (CBT) treatments for PTSD (Smyth, 1999). During these initial sessions, we discussed the concept of subjective units of distress (SUDs) and began evaluating his SUDs level at the beginning of sessions and periodically during sessions to acclimate him to these ratings prior to commencing gradual exposure.

Exposure-based treatments often result in increases of symptomatology, especially initially, necessitating the development of adaptive coping strategies for dealing with anxiety. Like many patients diagnosed with PTSD, Mr. Jones reported that he used avoidance strategies almost exclusively to deal with intrusive thoughts, flashbacks, and nightmares. Although these strategies are effective in providing relatively immediate decreases in anxiety, they play a role in the maladaptive negative reinforcement paradigm evident in PTSD and often socially isolate the individual. Thus, development of alternate and perhaps more adaptive mechanisms for coping are necessary. Sessions 4 and 5 focused on developing and practicing these requisite skills. During Session 4, a progressive muscle relaxation was introduced, and Mr. Jones participated in this fully and willingly. Given his chronic pain issues, he was encouraged to tense injured muscles briefly and lightly, as not to cause further injury. To further solidify the concept of SUDs and determine the effectiveness of relaxation in reducing anxiety, he rated his distress prior to and immediately after the relaxation exercise. This session was taped, and he practiced the relaxation method at home on three occasions outside of session. In addition, to address some of the cognitive components of PTSD, including but not limited to feelings of guilt, responsibility, and regret, assimilation and rational thinking were introduced as coping mechanisms. Straightforward didactic explanations, Socratic questioning, and use of metaphors were the primary therapeutic tools used to accomplish this. Mr. Jones acknowledged feelings of guilt and responsibility related to the traumatic events, although he was able to identify ways in which this was irrational. He stated that he recognized that he did not cause the individuals to engage him in a high-speed chase, and that he was not driving the vehicles that crashed. We explored the "damned if you do damned if you don't" dilemma that he was in, and he acknowledged that he could not be held responsible for the deaths of the individuals. We explored how he believed others, who did not know him, would perceive him. He noted that he believed that others would blame him and believe that he was responsible for the deaths of the individuals. We explored how this contributed to his self-worth and view of himself. It is interesting to note, he acknowledged these facets of his cognitive processes, although he did so in a formalized, detached fashion, as if he continued to hold these cognitive aspects at arm's length. This was addressed in later sessions.

The bulk of the remaining sessions (Sessions 6-11) was devoted to brief, gradual exposure to the traumatic events, as outlined by Smyth (1999). Exposure functioned to increase the client's emotional reactivity to the traumatic memory and related stimuli, such as somatic sensations that provoke panic and environmental stimuli that provoke phobic-like 
reactions. During sessions, brief exposure was alternated with relaxation exercises to encourage mastery of anxiety responses and demonstrate that anxiety can be managed and controlled by the client himself. Initially, an anxiety hierarchy was created for each of the traumatic events in terms of which aspects of the events elicited the highest level of anxiety. For the most part, the hierarchy was temporally sequential, in that as the events progressed in time, more anxiety was elicited by the memory. While creating hierarchies of the events, Mr. Jones reported moderate levels of anxiety, as measured by his SUDs ratings, although he omitted many details and again reported the events in a very logical, professional, disconnected fashion. Because of the multiple traumas, we decided to progress with exposure one event at a time, such that habituation to one trauma occurred prior to addressing the subsequent traumas. This approach would provide a level of continuity and conceptual clarity to sessions, as well as provide a potential secondary advantage of possible residual effect of exposure to the first event on the later exposure to the other traumas.

After creating anxiety hierarchies and identifying the first trauma that would be addressed, brief exposure commenced. During each session, an average of four brief exposures occurred to the trauma, alternating with relaxation exercises. For Sessions 7 and 8, Mr. Jones was instructed to visualize the events in his mind and provide a narrative of the events up until events rated as a 6 on his hierarchy. For this patient, this included describing patrolling in his police vehicle, turning on his lights and sirens, and then pursuing the car. The patient was instructed to hold this portion of the event in his mind while describing it. On average, the patient engaged in this description for 3 to 15 minutes, and then a brief relaxation exercise was initiated. Throughout the exposures and relaxation exercises, SUDs were taken frequently. During Sessions 7 and 8, the patient was instructed to inform the therapist if his SUDs exceeded 8. This did not occur. As the session progressed, the same stimuli and memories gradually failed to elicit the same levels of anxiety, and Mr. Jones was better able to relax during the relaxation segments. He also progressively provided more detail about the events with each exposure. During this session, the trauma narrative was audiotaped, and Mr. Jones was instructed to listen to the narrative at least 3 times each week between sessions.

During subsequent sessions (9 and 10), Mr. Jones continued gradual exposure, and with each successive session, he discussed details higher on his hierarchy. For Mr. Jones, this included discussing the car he was pursuing hitting another car, and him running to the vehicle to assist the passengers, and witnessing a badly injured and dismembered passenger. Mr. Jones also discussed the visual, tactile, and auditory images associated with this event, as well as his cognitive processes of feeling responsible and his own concerns with safety. He described in detail the process of giving CPR to the victim, including the tactile experience of feeling his broken bones and hearing his bones crack with each compression. During Session 9, he provided significantly more detail about the events, and stated that he was able to "see, smell, and feel" the stimuli related to the event. At one point, he stated that he felt physically nauseated because of picturing the image in his head. He was encouraged to stay with the image and was reassured that he could handle the anxiety and that he would not vomit. He did this successfully and then engaged in the relaxation response. In addition, as the sessions progressed, stimuli originally rated on his hierarchy as moderately distressing no longer elicited consistent anxiety. For example, during Session 9, Mr. Jones was asked to give an SUDs rating about a detail of the event, originally rated as a 6 on his 
hierarchy. He stated that it now was minimally anxiety provoking and rated at an SUDs of 2. He stated that he completed the homework assignment to listen to the audiotaped trauma narrative, although he stated that it was not anxiety provoking. For homework, he was instructed to listen to the trauma narrative at least 3 times, and to do at least two in-vivo exposures to the site of the incident. He was instructed to remain at the site until the anxiety diminished and should not leave until that reduction occurred. Although initially reticent, he agreed to complete the in-vivo exposure.

During the final session of brief, graduated exposure, the patient discussed the entire trauma from start to finish with minimal anxiety. As with the prior exposure sessions, brief exposures alternated with relaxation exercises. He noted the tactile, visual, and auditory stimuli associated with the traumatic event. Although he discussed the details of the events with little anxiety, he presented the information in a more connected, less detached fashion than during earlier sessions; that is, he did not report them in the same "police report" tone or style but appeared to have integrated these memories better, although they were not as anxiety provoking. His affect was notably less anxious, and he appeared much more relaxed during the narrative. He did not evidence behavioral signs (fidgeting, wringing his hands) of anxiety that were present in previous sessions. Again, stimuli previously rated as high on the initial hierarchy no longer elicited the same levels of anxiety, although Mr. Jones stated that he could "see them clearly in (his) mind's eye" and described them in great detail. During the relaxation segments, Mr. Jones guided himself in relaxation exercises to demonstrate mastery and reduce any possible dependency that may have developed with the therapist.

The objectives of Sessions 12 and 13 were to further reduce the client's emotional reactivity to the trauma-related stimuli by way of prolonged exposure exercises. Most of these sessions were spent in imaginal prolonged exposure and planning in-vivo exposure exercises. Strong desensitization effects are normally produced by this process, which should inoculate against relapse. During these sessions, Mr. Jones was encouraged to fully experience the trauma, without engaging in any anxiety-reduction techniques until instructed to do so. Mr. Jones was instructed to discuss all details of the event, without omitting any details, regardless of how distressing it became. The patient was instructed to visualize the event in his mind and focus in on the aspect that was most distressing. For this client, this included giving the victim CPR and seeing him trapped in the car. This event included cognitive, visual, auditory, and tactile aspects, such as hearing the popping of the victim's ribs and feeling the blood on his body. The therapist also worked to increase and maintain his level of anxiety by repeating the details of the events and the irrational beliefs that are associated with this event. The entire prolonged exposure lasted about 15 minutes. Initially, the patient became very anxious when focusing in on the aspect of the event. However, as the exposure progressed, his anxiety decreased despite focusing on the image itself. Over time, despite continued focus on the event and encouragement to "keep the anxiety up," the anxiety subsided. At the close of each session, the patient completed a relaxation exercise, even though his anxiety was already fairly low. Before leaving, the patient stated that he was "proud" of his progress, although he felt "exhausted."

By the end of the Session 13, the stimuli associated with the selected trauma were no longer eliciting any anxiety. Mr. Jones easily discussed the stimuli associated with the event, was readily engaging in the in-vivo homework assignments, and stated that he no longer avoided the intersection where the event occurred. Thus, the next two sessions were 
devoted to exposure to the other traumas that had occurred. Because of dramatic improvement that had occurred and based on the fact that there would likely be "carryover" progress from exposure to the first event, and because Mr. Jones requested this approach, prolonged exposure was used for the other traumas, as opposed to gradual exposure. During Session 14, Mr. Jones discussed an event that resulted in the death of the driver of the vehicle and all of the passengers. He described the visual, auditory, and cognitive aspects of this event in their entirety. He stated that this event was not as distressing as the other event because it did not involve the death of a child. He immediately proceeded to the aspects of the event that were rated as most distressing on his hierarchy and discussed those details until they no longer elicited anxiety. During this time, the therapist attempted to augment his level of anxiety by addressing relevant cognitive and visual aspects of the event. For example, the therapist encouraged Mr. Jones to focus on parts of the narrative that were particularly distressing and articulated various cognitive distortions (e.g., "It's your fault" and "You killed this person"). The patient was unable to maintain high levels of anxiety even in the presence of augmenting tactics, and the anxiety diminished to low SUDs levels within one session. He readily agreed to engage in in-vivo exposure exercises for homework and to discuss the traumas with his wife. The purpose of discussing the traumas with his wife was to encourage connectedness with her and to transfer some of the trusting therapeutic relationship developed from the therapist to other social support systems in Mr. Jones's life. During Session 14, prolonged exposure to the last event was conducted in a similar manner, with similar results. During this session, probes of the other traumas were conducted to determine levels of anxiety, and the patient stated that the most distressing images failed to evoke anxiety.

Session 15 was devoted to relapse prevention and review of progress during the course of treatment. This was accomplished by educating the client as to some common causes of relapse and how he can reduce the risk for relapse. Focal issues included exploring how prolonged stress from psychosocial factors, sleep deprivation, and substance abuse may lead to increases in symptomatology and the temptation to engage in maladaptive behaviors. Perhaps most important, the role of avoidance behaviors in potential relapse was addressed, specifically regarding how reverting back to avoidance, experiential and cognitive, facilitated relapse through reinforcement paradigms. We also discussed the skills that Mr. Jones had developed in terms of managing anxiety and highlighted his mastery of his symptoms through these skills. Mr. Jones acknowledged the dramatic changes that had occurred, and his pride in being able to make these changes. He also stated that he discussed the traumas with his wife, for the first time, and that he felt "more connected" with her than ever before. His affect during this session was dramatically different, in that he appeared happy, hopeful, and self-satisfied. It was also apparent that experiencing emotional connectedness with the therapist through exploration of his affective processes had resulted in a cognitive shift, specifically that expressing one's emotions was "allowable." In fact, he stated that he always believed that "men shouldn't talk" about these things, but "that's different now."

\section{Assessment of Progress}

As mentioned previously, throughout treatment progress was assessed through several measures. A steady decrease in general anxiety symptoms, as measured by the BAI, 
Figure 1

BDI-II and BAI Scores Over the Course of Baseline Sessions and Treatment

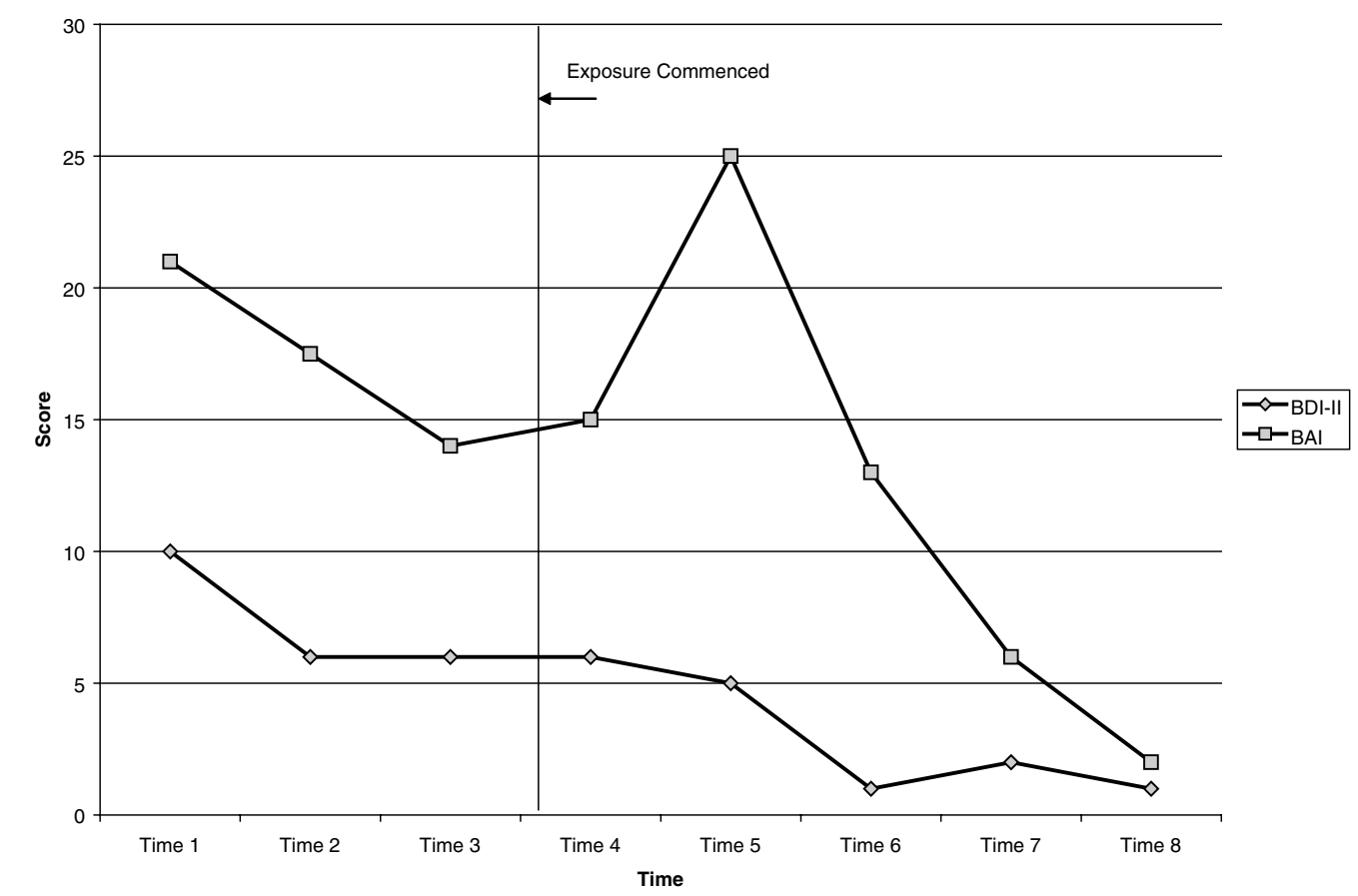

Note: BDI-II = Beck Depression Inventory 2nd edition; BAI = Beck Anxiety Inventory.

occurred throughout treatment, with minimal symptoms at the conclusion of treatment. Depressive symptoms were assessed by the BDI-II. Although Mr. Jones evidenced only mild symptoms at the onset of treatment, a decline in these symptoms occurred during the course of treatment as well (Figure 1).

The MPTSDSS was used to assess frequency and severity of PTSD symptoms. Again, a steady decline occurred in the frequency and severity of symptom clusters during the course of treatment, with no symptoms of numbness or intrusions, and minimal symptoms of avoidance or arousal (Figures 2 and 3).

In addition to these standardized measures, SUDs ratings were taken frequently throughout therapy. This was conducted, in part, to maintain a constant awareness of levels of distress and ensure that arousal was occurring during exposure sessions. From Figure 4, it appears that Mr. Jones was experiencing increases in anxiety during exposure and decreases following exposure during relaxation, as expected. This suggests that Mr. Jones was likely not using strategies to avoid arousal during exposure but rather was allowing himself to be exposed to the feared stimuli. In addition, SUDs ratings were obtained during the prolonged exposure sessions to determine if his level of distress would diminish over time to the most distressing stimuli. As seen in Figure 5, this appeared to have occurred, in that, even when he continued to visualize and discuss the distressing images, his anxiety diminished. 
Figure 2

Frequency of PTSD Symptom Clusters on the MPTSDSS

During the Course of Treatment

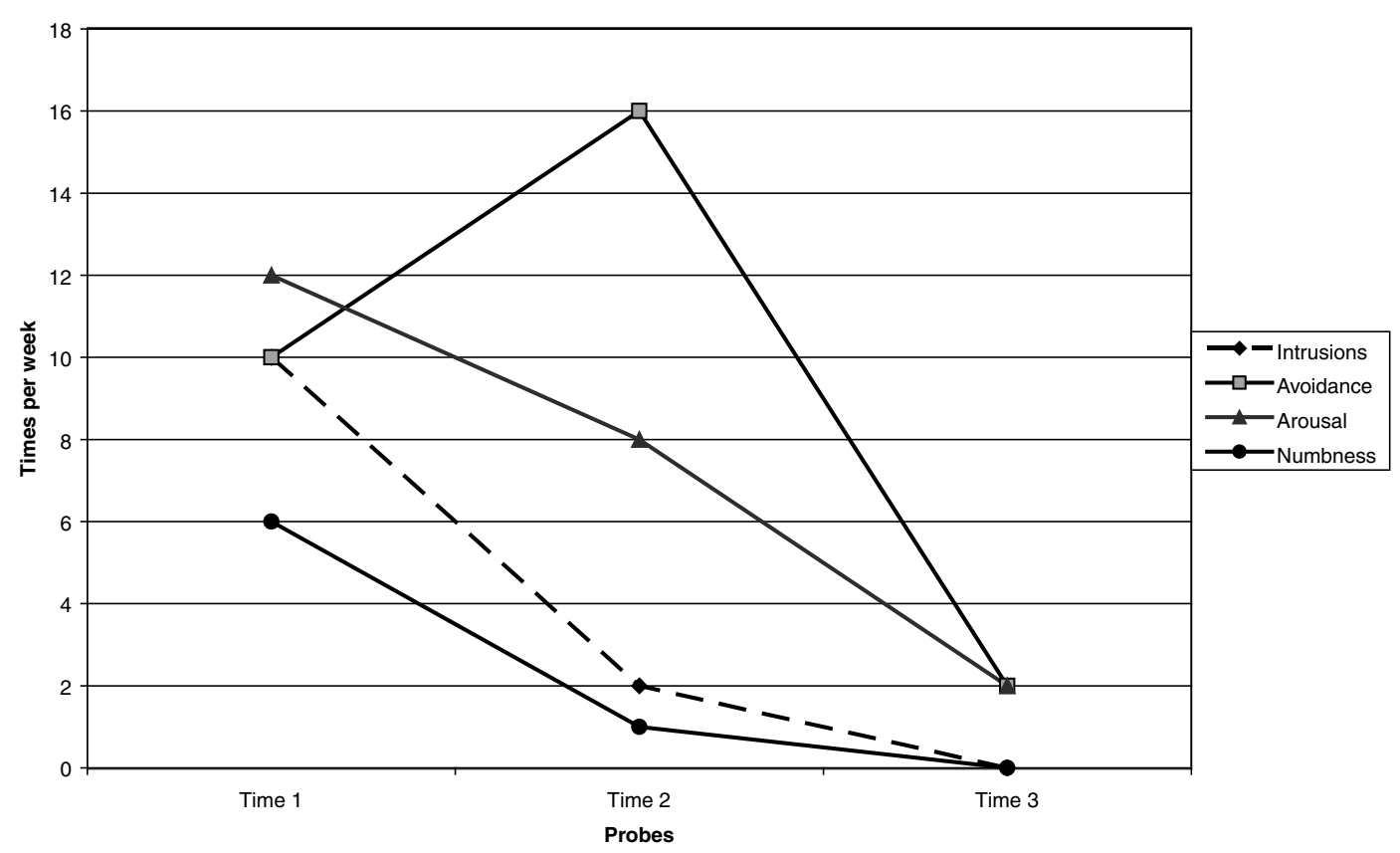

Note: PTSD $=$ posttraumatic stress disorder; MPTSDSS $=$ Modified PTSD Symptom Scale.

Based on these measures, it appears that great improvements occurred in terms of symptom reduction throughout treatment. In addition, in terms of his presentation, self-confidence, and self-efficacy, he made improvements as well. Although no systematic data in this regard were collected, during the course of treatment Mr. Jones more readily discussed his personal experiences than at the onset of treatment, grew to trust the therapist, and stated, at the end of therapy, that he was glad that he had revealed his emotional experiences to another person. This was a far cry from the reticent, controlled, and emotionally isolated client who initially presented for treatment. By completion of treatment, he discussed several other aspects of his emotional life, including his relationship with his parents, his experiences of racism as a police officer, and his relationship with his wife. This growth in his emotional functioning suggests that he may have abandoned some irrational and potentially maladaptive beliefs related to suppressing affective responses.

\section{Complicating Factors}

Several aspects of this case warranted special consideration. This client's distinctive and diverse experiences as an African American male in a predominately White geo- 


\section{Figure 3}

Severity of PTSD Symptom Clusters on the MPTSDSS on a 5-Point Likert-Type Scale During the Course of Treatment

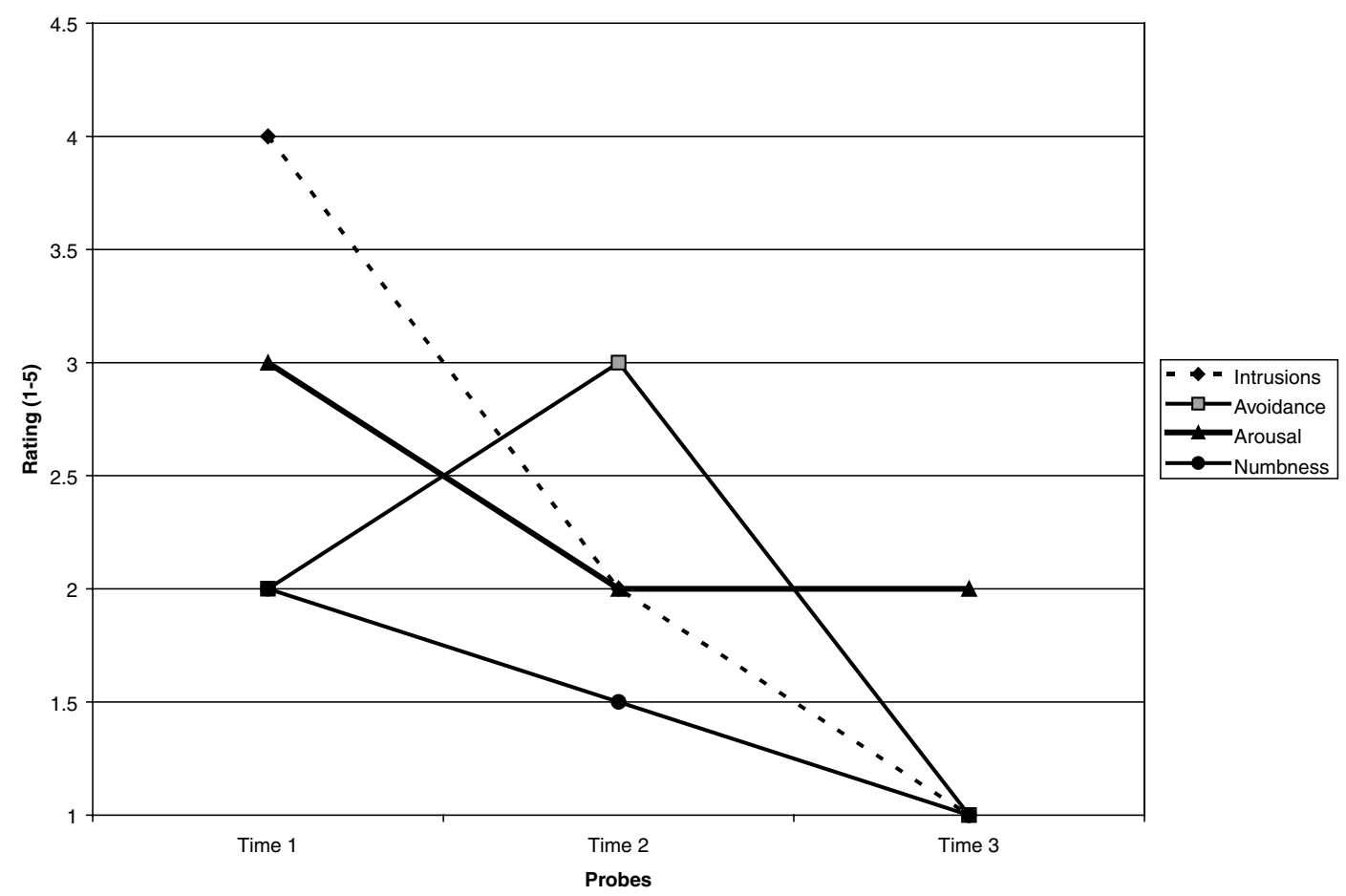

Note: PTSD = posttraumatic stress disorder; MPTSDSS = Modified PTSD Symptom Scale.

graphic area needed to be considered in treatment. Research has demonstrated that African Americans have been identified as a group that uses mental health services inconsistently (Okonji, Ososkie, \& Pulos, 1996; Thompson, Sanders, \& Bazile, 2004), and research suggests that minority groups utilize outpatient mental health services for psychiatric symptoms at approximately one half the rate of non-Hispanic White persons (L. A. Cooper et al., 2003). In addition, the data indicate that among racial minorities, rates of attrition are higher than among White patients. These data suggest that in this particular case, given that African American clients do not regularly seek treatment and, when they do, are more likely to stop attending prior to treatment completion, establishing a working, collaborative relationship with this client was an especially important aspect of treatment. In addition, exposure-based treatments require a collaborative therapeutic relationship coupled with the client perceiving the therapist as a trustworthy, understanding, and caring individual. Research has suggested that trust is a very important variable particularly with African American male clients. For minority clients, mistrust is a common barrier to mental health care (L. A. Cooper et al., 2003), and African American clients who rated their therapeutic relationship as high in mistrust were more likely to terminate therapy prematurely 
Figure 4

SUDs Ratings Prior to Exposure (Pre), Immediately After

Exposure (Post), and After Relaxation (Relax) for All Exposure

Exercises During Gradual Exposure Sessions

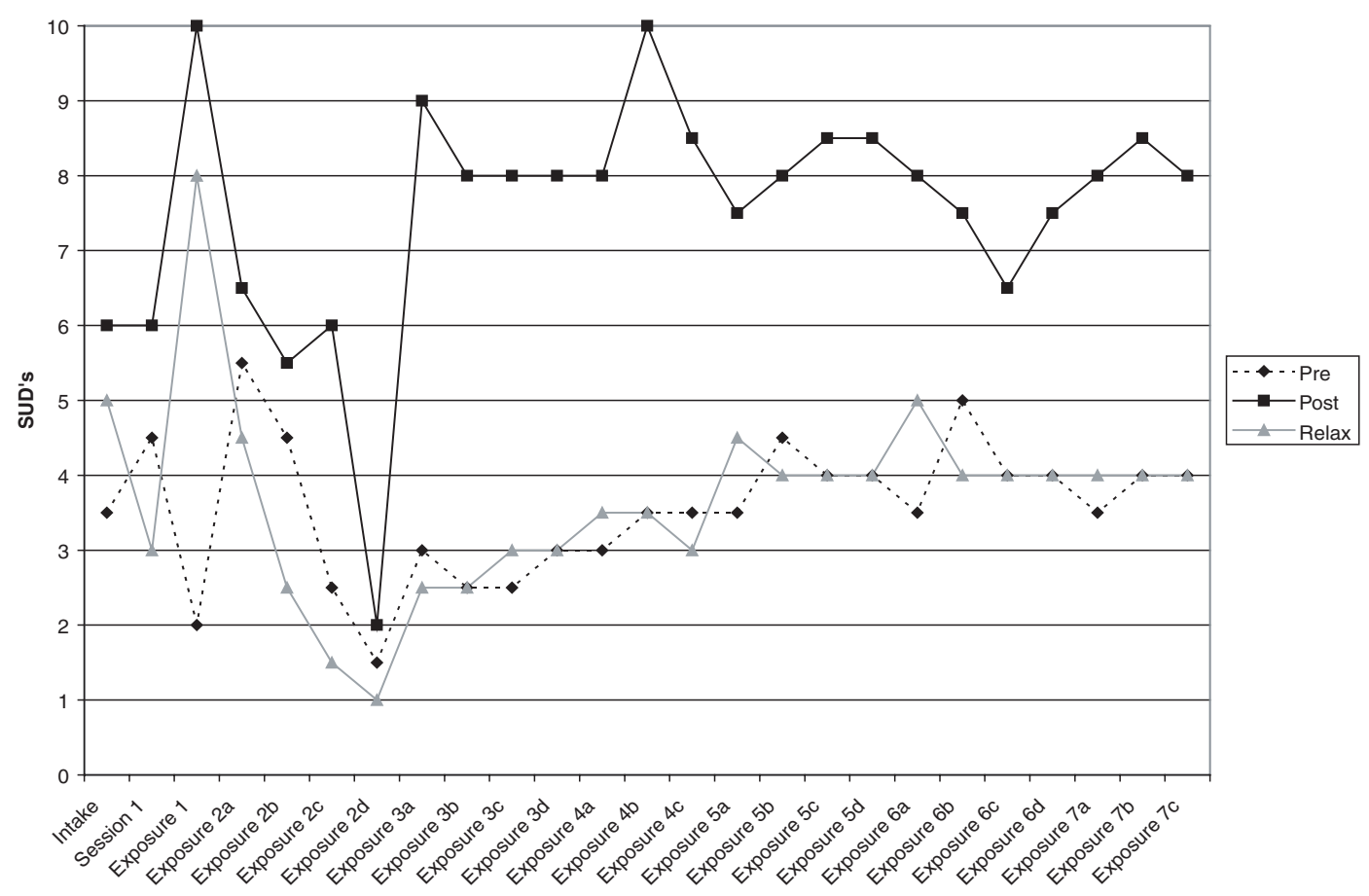

Note: SUDs = subjective units of distress.

(Thompson et al., 2004). Thus, given that the demographic characteristics of the client are associated with higher levels of mistrust in therapeutic relationships, this was an important area of focus for successful treatment. Other research has also suggested that PTSD symptoms, in some ethnic minority groups, can be exacerbated by experiences of racism. For this client, that was the case and, thus, was an important variable to consider. At various times throughout treatment, he noted racial discrimination and ethnic crimes directed at him and his family.

In addition to ethnic variables, the client's age and physical health were important treatment considerations. On the average, older adults tend to underutilize mental health services (Wetherell, 1998), so given the dual factors of ethnicity and chronological age, this patient likely was very reticent to seek mental health services and may have been more likely to stop treatment. In addition, other demographic variables may have played a role in this process, such as the age and gender of the therapist. Research suggests that male and female older patients preferred older therapists (Lauber \& Drevenstedt, 1993). Thus, given that the treating clinician was significantly younger than the client and appeared as such, this was an important factor considered during treatment and may have been a risk factor for premature termination of therapy. 


\section{Figure 5}

\section{SUDs Ratings During the First Prolonged Exposure Session}

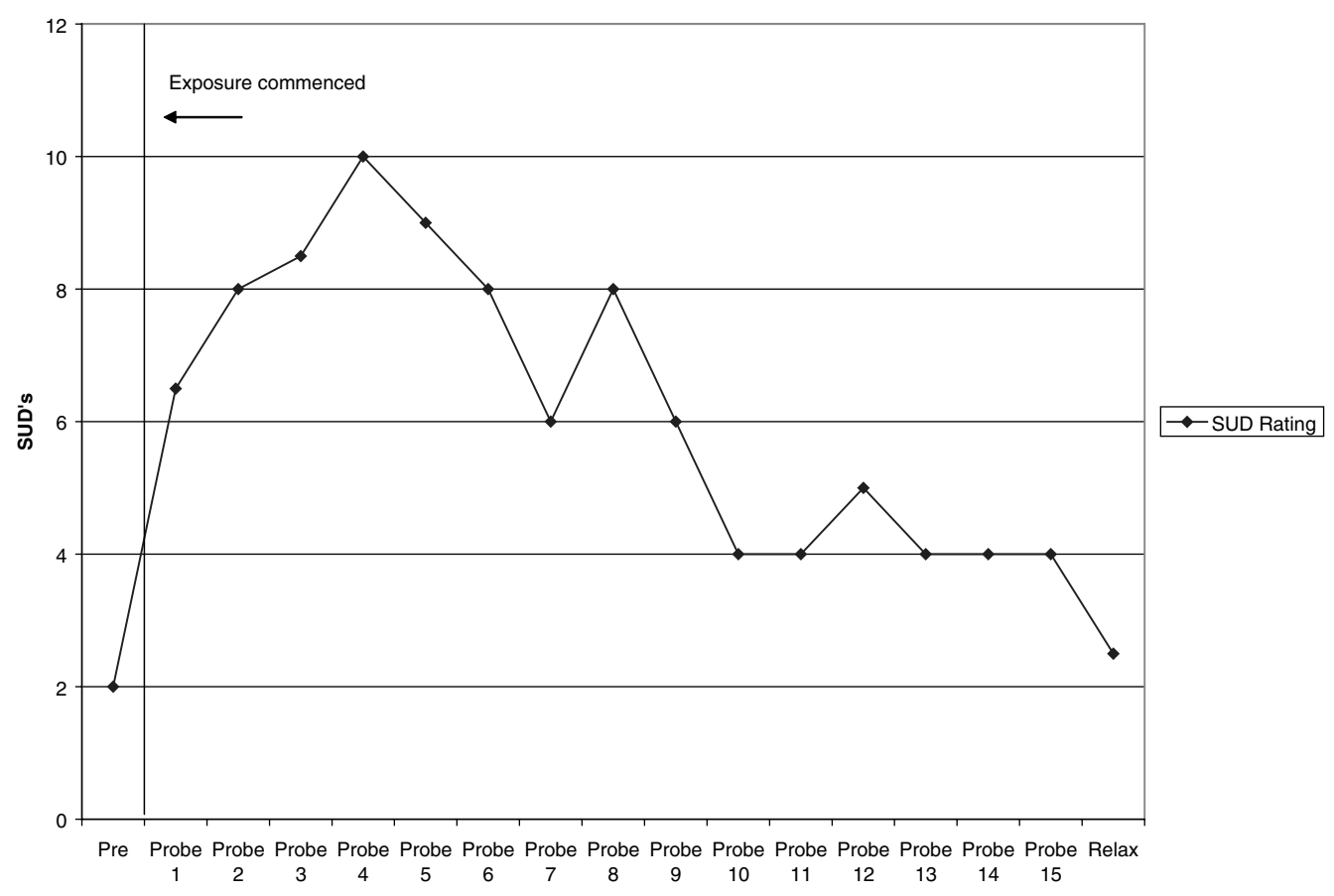

Note: SUDs = subjective units of distress.

\section{Managed Care Considerations}

Although treatment was conducted under auspices of the Department of Veterans Affairs, and thus, traditional managed care considerations are less applicable, such treatment could easily be applied in a setting where these concerns are more imminent. Cognitive-behavioral treatments are generally well received by managed care providers, in part because they often result in relatively quick improvements in symptomatology. Mr. Jones achieved significant improvement in his PTSD and mild depressive symptoms. Although a significant amount of time had transpired between traumatic events and the treatment, improvement was dramatic and achieved in a relatively brief period of treatment. This suggests that CBTs can be successfully applied to atypical cases of PTSD, not simply those where the trauma and treatment are in close proximity. Cognitive-behavioral treatments have the additional benefit of lower rates of relapse as compared to relapse rates of common psychotropic or anti-anxiety medications, resulting in lower health care costs in the long run.

\section{Follow-Up}

One follow-up session occurred approximately 8 weeks after completion of treatment. This session was conducted by the second author because the primary therapist had completed 
her training at the Medical Center. During this session, PTSD and possible depressive symptoms were discussed. The veteran reported significant improvement in overall functioning, no intrusive or hyperarousal symptoms, and stated that he was no longer engaging in avoidance behaviors. He completed the BDI-II and was asymptomatic. At this follow-up session, the patient indicated that he did not see the need for continued therapy because his functioning was dramatically improved and he was no longer experiencing any symptoms. Thus, improvements were maintained from treatment to a short-term follow-up. Unfortunately, no formal follow-up sessions were scheduled for longer term maintenance.

\section{Treatment Implications of the Case}

An overall implication of this case is that CBT is effective in ameliorating symptoms of PTSD and reducing depressive symptoms utilizing a gradual exposure methodology. This is consistent with the general effectiveness of CBTs for PTSD (Smyth, 1999) but also demonstrates that this approach can be effective when a long latency exists between the trauma and subsequent treatment. In addition, although police officers have long been recognized as a group that is likely to experience traumatic events and are at higher risk for developing PTSD (Harvey-Lintz \& Tidwell, 1997; Hickling \& Blanchard, 1999; Loo, 1986), most have focused on the effect of involvement or inflicting harm on others, rather than the effect of witnessing these events more passively. In this case, although Mr. Jones did not explicitly cause the deaths by shooting the victims, the same resulting feelings of guilt and responsibility occurred. As clinicians, we should be cognizant of the effect of these types of vicarious traumatizations that occur in law enforcement, and those in which the officer directly inflicts bodily harm. This case suggests that these cases are emotionally distressing, can lead to PTSD reactions, and can be successfully addressed using CBTs.

\section{Recommendations to Clinicians}

For PTSD, experiential and emotional avoidance is a central diagnostic and clinical feature. Patients presenting with these reactions often report pervasive and long-standing avoidance reactions. However, although a typical aspect of the clinical picture, this symptom cluster can hinder and, in fact, be iatrogenic to the treatment process. Particularly in cases where the latency between the trauma and treatment is large, these strategies of avoidance may be well-entrenched, well-rehearsed patterns of dealing with anxiety. As such, clinicians treating PTSD, particularly within an exposure paradigm, need to actively probe and assess the degree to which avoidance strategies are being utilized to numb the impact of the exposure exercises. Use of SUDs ratings, although perceived by some clinicians as intrusive and impersonal, is a vital aspect of this ongoing assessment. Because a key mechanism of action is the repeated exposure to feared stimuli that break the conditioning paradigm, the clinician must probe to ensure that the client is, in fact, experiencing full exposure to the stimuli, without utilizing anxiety-reduction mechanisms, such as avoidance. In this case, this was a primary reason why SUDs were used to such a great frequency; Mr. Jones was skilled at avoidance strategies, with regard to the trauma and interpersonal closeness with others. It would be vital to determine if the exposure sessions were, in fact, 
being numbed by specific strategies used to minimize their impact. SUDs data and the fact that Mr. Jones reported, on occasion, expected physiological reactions, such as nausea, suggested that he was experiencing a high level of exposure to the feared stimuli.

Exposure-based treatments have been shown generally to be highly efficacious (Barlow, 2002; Chambless et al., 1998; DeRubeis \& Crits-Christoph, 1998; Foa \& Meadows, 1997). However, to facilitate a client's active participation in the treatment, clinicians should attempt to match the client with the clinical strategy that is most likely to be acceptable to him or her. The concept of social validity is relevant here (Wolf, 1978), in that a treatment must be acceptable to the client for it to be successfully applied. In the case of anxiety reactions, prolonged exposure, or flooding, has been demonstrated to be effective in a relatively short period of time (Barlow, 2002; N. A. Cooper \& Clum, 1989; Foa \& Meadows, 1997; Keane, Fairbank, Caddell, \& Zimering, 1989). However, for many clients, despite the empirical evidence suggesting quick symptom relief, these types of approaches elicit too much distress and are unacceptable. A risk with flooding treatments is that the patient will drop out prematurely, which conceptually serves to amplify, rather than diminish, phobic reactions. Although it perhaps would have been more efficient and effective to proceed directly to the most anxiety-provoking stimuli, as noted on the hierarchy, a more graduated approach was taken to increase the social validity of the treatment. Thus, although seemingly less efficient, clinicians would often be well served to explore gradual exposure approaches, which are, while still distressing, often more acceptable to clients. In the case of Mr. Jones, given his demographic history and initial presentation that suggested that he might have stopped treatment prematurely, a gradual exposure treatment was likely a more acceptable clinical methodology. At the close of the last session, Mr. Jones completed a program satisfaction questionnaire, indicating that he believed the treatment to be highly effective, very useful, and stated that his goals were met and he had improved greatly. As occurred in this case, when the client has acclimated to gradual exposure and built a level of clinical rapport, prolonged exposure can be utilized. However, clinicians should be cautious in implementing prolonged methods from the onset and should reserve those interventions for later sessions or only in cases of clients who are very robust and who are very unlikely to stop therapy. In determining the appropriate treatment regime, clinicians should evaluate the empirical literature and the characteristics of the client to determine the course that is most likely to lead to improved functioning.

\section{References}

Barlow, D. H. (2002). Anxiety and its disorders: The nature and treatment of anxiety and panic (2nd ed.). New York: Guilford.

Beck, A. T., Brown, G., Epstein, N., \& Steer, R. A. (1988). An inventory for measuring clinical anxiety: Psychometric properties. Journal of Consulting and Clinical Psychology, 56(6), 893-897.

Beck, A. T., Steer, R. A., \& Brown, G. K. (1996). Beck Depression Inventory manual (2nd ed.). San Antonio, TX: The Psychological Corporation.

Carmin, C. N., \& Wiegartz, P. S. (2000). Successful and unsuccessful treatment of obsessive-compulsive disorder in older adults. Journal of Contemporary Psychotherapy, 30, 181-191.

Chambless, D. L., Baker, M. J., Baucom, D. G., Beutler, L. E., Calhoun, K. S., Crits-Christoph, P., et al. (1998). Update on empirically validated therapies II. Clinical Psychologist, 51, 3-16.

Cook, A. L., \& Shear, M. K. (2001). Treatment of a 50-year-old African American woman whose chronic posttraumatic stress disorder went undiagnosed for over 20 years. American Journal of Psychiatry, 158, 866-870. 
Cook, J. M. (2002). Traumatic exposure and PTSD in older adults: Introduction to the special issue. Journal of Clinical Geropsychology, 8, 149-152.

Cooper, L. A., Gonzales, J. J., Gallo, J. J., Rost, K. M., Meredith, L. S., Rubenstein, L. V., et al. (2003). The acceptability of treatment for depression among African American, Hispanic, and White primary care patients. Medical Care, 41, 479-489.

Cooper, N. A., \& Clum, G. A. (1989). Imaginal flooding as a supplementary treatment for PTSD combat veterans: A controlled study. Behavior Therapy, 3, 381-391.

DeRubeis, R. J., \& Crits-Christoph, P. (1998). Empirically supported individual and group psychological treatments for adult mental disorders. Journal of Consulting and Clinical Psychology, 66, 37-52.

Falsetti, S. A., Resnick, H. S., Resick, P. A., \& Kilpatrick, D. G. (1992, November). Posttraumatic stress disorder: The assessment of the frequency and severity of symptoms in clinical and nonclinical samples. Paper presented at the 26th annual convention of the Association for the Advancement of Behavior Therapy, Boston.

Foa, E. (1996). The efficacy of behavior therapy with obsessive compulsives. Clinical Psychologist, 49, 19-22.

Foa, E. \& Rothbaum, B. (1989). Behavioral psychotherapy for posttraumatic stress disorder. International Review of Psychiatry, 1, 219-227.

Foa, E. B., \& Meadows, E. A. (1997). Psychosocial treatments for posttraumatic stress disorder: A critical review. Annual Review of Psychology, 48, 449-480.

Harvey-Lintz, T., \& Tidwell, R. (1997). Effects of the 1992 Los Angeles civil unrest: Post traumatic stress disorder symptomatology among law enforcement officers. Social Science Journal, 34, 171-183.

Hickling, E. J., \& Blanchard, E. B. (Eds.). (1999). The international handbook of road traffic accidents and psychological trauma: Current understanding, treatment and law. Kidlington, Oxford, UK: Elsevier Science.

Keane, T. M., Fairbank, J. A., Caddell, J. M., \& Zimering, R. T. (1989). Implosive (flooding) therapy reduces symptoms of PTSD in Vietnam combat veterans. Behavior Therapy, 20, 245-260.

Lauber, B. M., \& Drevenstedt, J. (1993). Older adults preferences for age and sex of a therapist. Clinical Gerontologist, 14, 13-26.

Loo, R. (1986). Post-shooting stress reactions among police officers. Journal of Human Stress, 12, 27-31.

Maercker, A. (2002). Life-review technique in the treatment of PTSD in elderly patients: Rationale and three single case studies. Journal of Clinical Geropsychology, 8, 239-249.

Mowrer, O. H. (1960). Learning theory and behavior. Oxford, England: Wiley.

Okonji, J. M. A., Ososkie, J. N., \& Pulos, S. (1996). Preferred style and ethnicity of counselors by African American males. Journal of Black Psychology, 22, 329-339.

Smyth, L. (1999). Overcoming post traumatic stress disorder. Oakland, CA: New Harbinger.

Solomon, Z. (1992). The "Koach" project for treatment of combat-related PTSD: Rationale, aims, and methodology. Journal of Traumatic Stress, 5, 175-193.

Steer, R. A., Ball, R., Ranieri, W. F., \& Beck, A. T. (1997). Further evidence for the construct validity of the Beck Depression Inventory-II with psychiatric outpatients. Psychological Reports, 80, 443-446.

Thompson, V. L., Sanders, V. L., \& Bazile, A. A. (2004). African Americans' perceptions of psychotherapy and psychotherapists. Professional Psychology: Research and Practice, 35, 19-26.

Waller, N. G. (1998). Review of the Beck Depression Inventory-1993 Revised. In J. C. Impara \& B. S. Plake (Eds.), The thirteenth mental measurements yearbook (pp. 120-121). Lincoln, NE: Buros Institute of Mental Measurements.

Wetherell, J. L. (1998). Treatment of anxiety in older adults. Psychotherapy, 35, 444-455.

Wolf, M. M. (1978). Social validity: The case for subjective measurement or how applied behavior analysis is finding its heart. Journal of Applied Behavior Analysis, 11, 203-314.

Tara L. Cornelius completed her PhD in clinical psychology at Western Michigan University and is currently an assistant professor at Grand Valley State University. Her current research interests include dyadic communication processes in intimate relationships, interpersonal violence, and psychopathological functional processes in anxiety disorders.

Rita Kenyon-Jump, $\mathrm{PhD}$, is a clinical psychologist with the Department of Veterans Affairs Medical Center in Battle Creek, Michigan. She is the Military Sexual Trauma Coordinator for seven Veterans Affairs (VA) Medical Centers in Michigan, Illinois, and Indiana and currently serves on a National Committee for Women Veterans Mental Health. 